\section{Kidney \\ Blood Pressure Research}

\title{
Risk Factor Analysis for AKI Including Laboratory Indicators: a Nationwide Multicenter Study of Hospitalized Patients
}

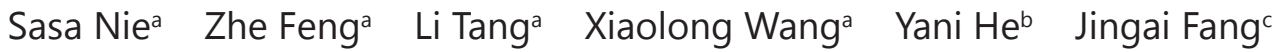 \\ Suhua Lid Yibin Yange Huijuan Mao ${ }^{f}$ Jundong Jiaog ${ }^{g}$ Wenhu Liu ${ }^{h}$ Ning Cao \\ Wenge Wang j Jifeng Sun ${ }^{k}$ Fengmin Shao' Wenge Lim ${ }^{m}$ Qiang He ${ }^{n}$ \\ Hongli Jiang ${ }^{0}$ Hongli Lin ${ }^{p}$ Ping Fu ${ }^{q}$ Xinzhou Zhangr Yinghong Liu ${ }^{5}$ \\ Yonggui Wu ${ }^{t}$ ChunSheng Xi ${ }^{u}$ Meng Liang ${ }^{v}$ Zhijie Quw Jun Zhu ${ }^{x}$ Guangli Wu \\ Yali Zheng ${ }^{z}$ Yu Na ${ }^{A}$ Ying Li ${ }^{B}$ Wei Lic Guangyan Cai ${ }^{\mathrm{C}}$ Xiangmei Chen ${ }^{\mathrm{a}}$
}

aDepartment of Nephrology, Chinese PLA General Hospital, Chinese PLA Institute of Nephrology, State Key Laboratory of Kidney Diseases, National Clinical Research Center for Kidney Diseases, Beijing, ${ }^{b}$ Department of Nephrology, Daping Hospital, the Third Military Medical University, Chongqing, 'Department of Nephrology, the First Hospital of Shanxi Medical University, Taiyuan, Shanxi, dDepartment of Nephrology, the First Affiliate Hospital of Xinjiang Medical University, Urumqi, Xinjiang, e'Department of Nephrology, Affiliated Hospital of Zunyi Medical University, Zunyi, Guizhou, 'Department of Nephrology, the Jiangsu Province Hospital, Nanjing, Jiangsu, פDepartment of Nephrology, the Second Affiliated Hospital of Harbin Medical University, Harbin, Heilongjiang, 'Department of Nephrology, Beiijng Freindship Hospital, Capital Medical University, Beijing, iDepartment of blood purification, General Hospital of Shenyang Military Area Command, Shenyang, Liaoning, 'Department of Nephrology, Lanzhou University Second hospital, Lanzhou, Gansu, kDepartment of Nephrology, Tangdu Hospital, The Fourth Military Medical University, Xi'an, Shanxi, 'Department of Nephrology, Henan Provincial People's Hospital, Zhengzhou University, Zhengzhou, Henan, ${ }^{m}$ Department of Nephrology, China-Japan Friendship Hospital, Beijing, "Department of Nephrology, Zhejiang Provincial People's Hospital, Hangzhou, Zhejiang, 'Blood purification center, the First Affiliated Hospital of Xian Jiaotong University, Xi'an, PDepartment of Nephrology, the First Affiliated Hospital of Dalian Medical University, Dalian, Liaoning, aDepartment of Nephrology, West China Hospital of Sichuan University, Chengdu, Sichuan, 'Department of Nephrology, Shenzhen People's Hospital, Shenzhen, Guangdong, sDepartment of Nephrology, the Second Xiangya Hospital, Central South University, Changsha, Hunan, 'Department of Nephrology, the Frist Affiliated Hospital of Anhui Medical university, Hefei, Anhui, "Department of Nephrology, the Lanzhou Military General Hospital, Lanzhou, Gansu, vDepartment of Nephrology, Chenggong Hospital Xiamen University, Xiamen, Fujian, wDepartment of Nephrology, the Second Hospital of Jilin University, Changchun, Jilin, ×Department of Nephrology, the Chengdu Military General Hospital, Chengdu, Sichuan, yDepartment of Nephrology, the Bethune international peace hospital of PLA, Shijiazhuang, Hebei, 'Department of Nephrology, Ningxia People's Hospital, Yinchuan, Ningxia, ${ }^{A}$ Department of Nephrology, the 306th

Nie S. and Feng Z. contributed equally to this work and therefore share first authorship. 


\title{
Kidney \\ Blood Pressure Research
}

Nie et al.: Risk Factors for AKI Including Laboratory Indicators

Hospital of PLA, Beijing, BDepartment of Nephrology, the Third Hospital of Hebei Medical University, Shijiazhuang, Hebei, 'Department of Nephrology, Affiliated Hospital of Shandong University of Traditional Chinese Medicine, Jinan, Shandong, China

\section{Key Words}

Acute kidney injury • Epidemiology • Mortality • Risk factors • Serum uric acid

\begin{abstract}
Background/Aims: Risk factor studies for acute kidney injury (AKI) in China are lacking, especially those regarding non-traditional risk factors, such as laboratory indicators. Methods: All adult patients admitted to 38 tertiary and 22 secondary hospitals in China in any one month between July and December 2014 were surveyed. AKI patients were screened according to the Kidney Disease: Improving Global Outcomes' definition of AKI. Logistic regression was used to analyze the risk factors for AKI, and Cox regression was used to analyze the risk of in-hospital mortality for AKI patients; additionally, a propensity score analysis was used to reconfirm the risk factors among laboratory indicators for mortality. Results: The morbidity of AKI was $0.97 \%$. Independent risk factors for AKI were advancing age, male gender, hypertension, and chronic kidney disease. All-cause mortality was $16.5 \%$. The predictors of mortality in AKI patients were advancing age, tumor, higher uric acid level and increases in Acute Physiologic Assessment and Chronic Health Evaluation II and Sequential Organ Failure Assessment scores. The hazard ratio (HR) for mortality with uric acid levels > $9.1 \mathrm{mg} / \mathrm{dl}$ compared with $\leq 5.2 \mathrm{mg} / \mathrm{dl}$ was 1.78 (95\% CI: 1.23 to 2.58) for the AKI patients as a group, and was 1.73 (95\% CI: 1.24 to 2.42) for a propensity score-matched set. Conclusion: In addition to traditional risk factors, uric acid level is an independent predictor of all-cause mortality after AKI.
\end{abstract}

(C) 2017 The Author(s)

Published by S. Karger AG, Basel

\section{Introduction}

Acute kidney injury (AKI) is a common critical disorder in hospitalized patients that carries a high risk of mortality. A meta-analysis in 2015 involving more than 700 studies revealed an AKI morbidity rate for hospitalized patients of $21 \%$, and a $21 \%$ mortality rate among AKI patients; furthermore, it indicated that the prognosis of AKI had not significantly improved [1]. The risk of developing chronic kidney disease (CKD) among AKI patients was 8.8 times higher than the risk among non-AKI patients; similarly, the risk of progression to end-stage renal disease (ESRD) was 3.1 times higher, and the risk of mortality was 2 times higher [2]. The pathogenic mechanism of AKI remains unclear at present. Because the Kidney Disease: Improving Global Outcomes (KDIGO) guidelines do not recommend any drug for the prevention and treatment of AKI [3], therapy still relies on long-standing symptomatic and supportive treatment. However, AKI is to some extent preventable, controllable, and even reversible with timely intervention. Therefore, it is particularly important to identify risk factors that predispose individuals to develop AKI and that predict mortality in those with AKI. However, existing risk factors mainly focus on specific susceptibilities and certain demographic characteristics, comorbidities, and treatments, and they ignore non-traditional risk factors, especially laboratory indicators.

In this study, we combined data from 38 tertiary hospitals and 22 secondary hospitals in China to conduct a large-scale, multidisciplinary, cooperative AKI study of adult hospitalized patients. This study covered 21 of 31 provinces, autonomous regions, and direct-controlled 


\section{Kidney Blood Pressure Research}

Nie et al.: Risk Factors for AKI Including Laboratory Indicators

municipalities in China and 79\% of the Chinese population. The purpose was to explore risk factors that affect the development of AKI and mortality in AKI patients.

\section{Materials and Methods}

\section{Study Design and Population}

We selected 38 representative tertiary hospitals and 22 secondary hospitals in 21 provinces, autonomous regions, and direct-controlled municipalities in mainland China to conduct a nationwide, multicenter, cross-sectional study based on the sampling method. All patients $\geq 18$ years old who were hospitalized in any one month between July $1^{\text {st }}$ and December $31^{\text {st }}$ of 2014 were included. Through case analysis, AKI patients who were hospitalized for $>24 \mathrm{~h}$ and diagnosed and treated for the first time were screened according to the AKI clinical practice guidelines released by KDIGO in 2012. Patients undergoing maintenance hemodialysis (including peritoneal dialysis) and those with CKD stage 5 without dialysis, kidney organ transplantation, and nephrectomy were excluded. This study was approved by the Ethics Committee of People's Liberation Army (PLA) General Hospital and other participating institutions in China.

\section{Data Sources}

Information for the hospitalized patients, including gender, age, underlying disease, survival status at discharge, and length of hospital stay, was collected. Demographic data, underlying and concomitant disease, laboratory indicators within $24 \mathrm{~h}$ of AKI diagnosis, injury factors for AKI development, use of suspected nephrotoxic drugs, AKI etiology and renal replacement therapy (RRT) parameters were retrieved from the AKI on-line electronic medical record registration system (http://pd.cnrds.net:6780/aki/user. do?action=firstpage). AKI was defined as an increase in creatinine of more than $26.5 \mu \mathrm{mol} / \mathrm{l}(0.3 \mathrm{mg} / \mathrm{dl})$ within $48 \mathrm{~h}$ or a confirmed/speculated increase in creatinine of 1.5 -fold over baseline within $7 \mathrm{~d}$. The AKI stage was determined by the highest creatinine level after AKI development [3]. Underlying and concomitant diseases were determined by the ICD-10 codes assigned at discharge. CKD was determined based on the diagnostic coding, history of renal injury more than 3 months prior to admission, or an estimated glomerular filtration rate (eGFR) $<60 \mathrm{~mL} / \mathrm{min} / 1.73 \mathrm{~m}^{2}$ (calculated based on the Modification of Diet in Renal Disease (MDRD) formula) for more than 3 months [4]. Injury factors and causes were confirmed by two trained senior physicians in the Department of Nephrology and were based on patient records. An AKI case was considered drug-related if it met one of the following criteria: the diagnosis obtained from renal biopsy was drug-related AKI or the patient received nephrotoxic drugs more than 3 days before the creatinine change and no other apparent causes were discovered [5]. For patients who had received more than one type of RRT, as long as continuous RRT (CRRT) was included, then CRRT was considered as the predominant mode in the study [6]. Sustained low-efficiency dialysis (SLED) was determined by the use of extended duration RRT (6-18 h), in which conventional hemodialysis machines with reduced dialysate and blood flow rates were used [7]. The dialysate and blood flow rates were all $100-300 \mathrm{~mL} / \mathrm{min}$.

\section{Statistical Analysis}

SPSS 22.0 software was used for the statistical analysis (SPSS, Inc., Chicago, IL, USA). Variables are expressed as the mean $\pm S D$, median $\left(Q u, Q_{L}\right)$, or $n(\%)$, as appropriate. Comparisons between two groups were performed using the $t$ test, Mann-Whitney $U$ test, or Pearson $\chi^{2}$ test, as appropriate. Univariate logistic regression analysis was performed for all factors that could cause AKI, including demographic data, and underlying disease. Additionally, a collinearity diagnosis was performed. The variables that satisfied statistical significance $(\mathrm{P}<0.05)$ in univariate logistic regression analysis and showed no collinearity with others were used for the multivariate logistic regression analysis. Univariate Cox regression analysis was performed on all factors that could affect the mortality of AKI patients, including demographic data, AKI stage, underlying disease, concomitant disease, injury factors, heart rate, mean arterial pressure (MAP), laboratory indicators, Acute Physiologic Assessment and Chronic Health Evaluation II (APACHE II) score, Sequential Organ Failure Assessment (SOFA) score, and RRT. The same method was used to perform multivariate Cox regression analysis. To reconfirm the laboratory indicators that constituted risk factors, a propensity score was performed between the survivors and non-survivors among AKI patients using 1:1 


\section{Kidney Blood Pressure Research}

Table 1. Comparison of characteristics between AKI and non-AKI groups

\begin{tabular}{|c|c|c|c|c|}
\hline Variables & $\begin{array}{c}\text { AKI } \\
(n=1845)\end{array}$ & $\begin{array}{c}\text { Non-AKI } \\
(\mathrm{n}=189190)\end{array}$ & $\begin{array}{c}\text { All }(\mathrm{n}=191035) \\
(\mathrm{n}=189,190) \text { patients }(\mathrm{n}=191,035)\end{array}$ & $P$ value \\
\hline Age (years) & $\begin{array}{c}60.5 \pm 17.4 \\
1254 \\
(68.0 \%)\end{array}$ & $53.1 \pm 17.0$ & $53.1 \pm 17.0$ & $<0.001^{*}$ \\
\hline Men & & $\begin{array}{c}94346 \\
(49.9 \%)\end{array}$ & $95600(50.0 \%)$ & $<0.001 *$ \\
\hline Regional distribution & & & & $<0.001^{*}$ \\
\hline South Central & $124(6.7 \%)$ & $\begin{array}{c}25026 \\
(13.2 \%)\end{array}$ & $25150(13.2 \%)$ & \\
\hline Northeast & $\begin{array}{c}246 \\
(13.3 \%)\end{array}$ & $\begin{array}{c}39031 \\
(20.6 \%)\end{array}$ & $39277(20.6 \%)$ & \\
\hline Northwest & $\begin{array}{c}397 \\
(21.5 \%)\end{array}$ & $\begin{array}{c}38060 \\
(20.1 \%)\end{array}$ & 38457 (20.1\%) & \\
\hline East & $\begin{array}{c}233 \\
(12.6 \%)\end{array}$ & $\begin{array}{c}20116 \\
(10.6 \%)\end{array}$ & $20349(10.7 \%)$ & \\
\hline North & $\begin{array}{c}354 \\
(19.2 \%)\end{array}$ & $\begin{array}{c}29558 \\
(15.6 \%)\end{array}$ & $29912(15.7 \%)$ & \\
\hline Southwest & $\begin{array}{c}491 \\
(26.6 \%)\end{array}$ & $\begin{array}{c}37399 \\
(19.8 \%)\end{array}$ & $37890(19.8 \%)$ & \\
\hline \multicolumn{5}{|l|}{ Underlying diseases } \\
\hline Hypertension & $\begin{array}{c}772 \\
(41.8 \%)\end{array}$ & $\begin{array}{c}38900 \\
(20.6 \%)\end{array}$ & $39672(20.8 \%)$ & $<0.001^{*}$ \\
\hline Diabetes & $\begin{array}{c}329 \\
(17.8 \%)\end{array}$ & $\begin{array}{c}21277 \\
(11.2 \%)\end{array}$ & $21606(11.3 \%)$ & $<0.001^{*}$ \\
\hline $\begin{array}{l}\text { Cardiovascular } \\
\text { disease }\end{array}$ & $\begin{array}{c}457 \\
(24.8 \%)\end{array}$ & $\begin{array}{c}31642 \\
(16.7 \%)\end{array}$ & $32099(16.8 \%)$ & $<0.001^{*}$ \\
\hline $\begin{array}{l}\text { Chronic liver } \\
\text { disease }\end{array}$ & $89(4.8 \%)$ & $10105(5.3 \%)$ & $10194(5.3 \%)$ & 0.33 \\
\hline COPD & $121(6.6 \%)$ & $6579(3.5 \%)$ & $6700(3.5 \%)$ & $<0.001^{*}$ \\
\hline CKD & $\begin{array}{c}187 \\
(10.1 \%) \\
\end{array}$ & $10533(5.6 \%)$ & $10720(5.6 \%)$ & $<0.001^{*}$ \\
\hline AKI, acute kidne & $\begin{array}{l}\text { ariables were } \\
\text { injury; COPD, }\end{array}$ & $\begin{array}{r}\text { xpressed with } \mathrm{m} \\
\text { hronic Obstructi } \\
* \mathrm{P}<\end{array}$ & $\begin{array}{l}\mathrm{SD} \text {, median }\left(\mathrm{Qu}_{\mathrm{U}}, \mathrm{Q}_{\mathrm{L}}\right) \text { or } \mathrm{n}(\%) . \\
\text { nonary Disease; } \mathrm{CKD} \text {, Chronic Kidr }\end{array}$ & ease \\
\hline
\end{tabular}

matching. The matched variables included other data, in addition to laboratory indicators. A multivariate Cox regression analysis was then used to evaluate independent risk factors in matched patients. All $\mathrm{p}$ values were two-sided. $\mathrm{P}<0.05$ was defined as statistically significant.

\section{Results}

The 191, 035 hospitalized patients had a mean age of $53.1 \pm 17.0$ years. The male-tofemale ratio was 1.0: 1.0. Among these patients, 1845 were diagnosed with AKI, and the morbidity rate was $0.97 \%$. The mean age of the AKI patients was higher than that of the nonAKI patients $(60.5 \pm 17.4$ vs. $53.1 \pm 17.0, \mathrm{P}<0.001)$, and the AKI patients included a higher percentage of males ( $68 \%$ vs. $49.9 \%, \mathrm{P}<0.001)$.

\section{Risk Factors for AKI Development}

Table 1 shows the risk factors related to AKI development. The univariate regression analysis revealed that advancing age, male gender, and the presence of hypertension, diabetes, cardiovascular disease, chronic obstructive pulmonary disease (COPD) and CKD were risk factors for AKI development. The independent risk factors associated with AKI 


\section{Kidney Blood Pressure Research}

Table 2. Factors associated with AKI development according to multivariate logistic regression analysis

\begin{tabular}{ccc}
\hline Variables & OR $(95 \% \mathrm{CI})$ & P value \\
\hline Sex (male* vs female) & $0.52(0.47,0.57)$ & $<0.001^{\dagger}$ \\
Age (per 10 years increase) & $1.18(1.15,1.22)$ & $<0.001^{\dagger}$ \\
Underlying diseases (yes vs no*) & & \\
Hypertension & $2.16(1.94,2.40)$ & $0.001^{\dagger}$ \\
Diabetes & $1.09(0.96,1.24)$ & 0.18 \\
Cardiovascular disease & $0.99(0.88,1.11)$ & 0.86 \\
COPD & $1.18(0.97,1.43)$ & 0.10 \\
CKD & $1.42(1.21,1.66)$ & $<0.001^{\dagger}$ \\
\hline
\end{tabular}

All variables listed in the table were included in the logistic regression model and adjusted for regional distribution. AKI, acute kidney injury; COPD, Chronic Obstructive Pulmonary Disease; CKD, Chronic Kidney Disease *Reference value; $\nmid \mathrm{P}<0.05$.

development are presented in Table 2. The multivariate logistic regression analysis revealed advancing age, male gender, hypertension and CKD as risk factors for AKI development.

\section{Epidemiological Characteristics of AKI Patients (Table 3)}

Among the 1845 AKI patients, the male-to-female ratio was 2.12: 1.00. Of these AKI patients, 1148 (62.2\%), 372 (20.2\%), 325 (17.6\%) were Stage I, stage II, and stage III, respectively. The top three diseases underlying AKI were hypertension (41.8\%), coronary heart disease (19.6\%), and diabetes (17.8\%). Infection $(46.6 \%)$ was the most common risk factor, followed by drug use (35.9\%). The mean hemoglobin level of the patients who were diagnosed with AKI within $24 \mathrm{~h}$ was $11.5 \pm 2.9 \mathrm{~g} / \mathrm{dl}$, which was lower than that of healthy individuals. The albumin $(3.3 \pm 0.8 \mathrm{~g} / \mathrm{dl})$ level was also slightly lower. In terms of disease severity, the APACHE II score was $11(7,16)$, and the SOFA score was $5(2,9)$. Of the AKI patients, 73 (4.0\%) had multiple organ dysfunction syndrome (MODS), and 82 (4.4\%) had sepsis. Most of the cases were due to pre-renal etiology, followed by renal etiology; post-renal etiology accounted for less than $10 \%$ of the cases. There were 257 cases of drug-related AKI (Table 4), comprising $13.9 \%$ of the AKI patients. Antibiotics-induced AKI accounted for almost half of the cases $(48.2 \%, 124$ cases). One hundred forty-nine $(8.1 \%)$

Table 3. Epidemiological and clinical characteristics of survivors and non-survivors among AKI patients Variables were expressed with mean $\pm S D$, median $\left(Q_{U}, Q_{L}\right)$ or $n(\%)$.Conversion factor for total bilirubin, direct bilirubin, serum creatinine, and serum uric acid in $\mathrm{mg} / \mathrm{dL}$ to $\mu \mathrm{mol} / \mathrm{L}, \times 17.1, \times 17.1, \times 88.40, \times 59.48$, respectively; Conversion factor for urea nitrogen, serum glucose, serum calcium, and serum phosphate in $\mathrm{mg} / \mathrm{dL}$ to $\mathrm{mmol} / \mathrm{L}, \times 0.357, \times 0.05551, \times 0.2495, \times 0.3229$, respectively. AKI, acute kidney injury; COPD, Chronic Obstructive Pulmonary Disease; CKD, Chronic Kidney Disease; MODS, Multiple Organ Dysfunction Syndrome; CPR, cardiopulmonary resuscitation; BMI, Body Mass Index; MAP, mean arterial pressure; DIC, Disseminated Intravascular Coagulation; Hb, hemoglobin; WBC, white blood cell; PLT, platelet count; TP, total protein; ALB, albumin; ALT, alanine aminotransferase; AST, aspartate aminotransferase; TBil, total bilirubin; DBiL, direct Bilirubin; SCr, serum creatinine; BUN, blood urea nitrogen; SUA, Serum uric acid; APACHE, Acute Physiology and Chronic Health Evaluation; SOFA, Sequential Organ Failure Assessment; RRT, renal replacement therapy. Hypovolemia: according to the judgment of the clinical physicians, a reduced effective blood volume induced by a variety of causes that cannot maintain the normal blood supply and oxygen supply for tissues in the body and a systolic blood pressure $\leq 90 \mathrm{mmHg}$ for more than $1 \mathrm{~h} \mathrm{[37].}$ Low cardiac output: insufficient cardiac systolic function with various causes, a reduction of systemic blood pressure ( $\leq 90 \mathrm{mmHg}$ ), and concomitant tissue hypoxia syndrome [37]. Massive blood transfusion: transfusion of more than 10 units of red blood cells within a short time frame (within 72 h) [37]. Post - CPR: patients lose autonomic circulation and pulse due to cardiopulmonary respiratory and circulatory system collapse; therefore, the patients require CPR, defibrillation, or adrenaline to save their lives and survive for more than $24 \mathrm{~h}$ [37]. Rhabdomyolysis: serum creatine kinase $>5000 \mathrm{U} / \mathrm{L}$ or plasma myoglobin $>5000 \mu \mathrm{g} / \mathrm{L}$ [37]. *P $<0.05$ 


\section{Kidney \\ Blood Pressure Research}

Kidney Blood Press Res 2017;42:761-773

DOI: 10.1159/000484234

Nie et al.: Risk Factors for AKI Including Laboratory Indicators

\begin{tabular}{|c|c|c|c|c|}
\hline Variables & Survivors $(\mathrm{n}=1540)$ & Non-survivors $(\mathrm{n}=305)$ & All $(n=1845)$ & $\mathrm{P}$ value \\
\hline Age (years) & $59.8 \pm 17.3$ & $64.5 \pm 17.7$ & $60.5 \pm 17.4$ & $<0.001^{*}$ \\
\hline Men & $1046(67.9 \%)$ & $208(68.2 \%)$ & $1254(68.0 \%)$ & 0.93 \\
\hline Regional distribution & & & & $<0.001^{*}$ \\
\hline Central and southern & $110(7.2 \%)$ & $14(4.6 \%)$ & $124(6.8 \%)$ & \\
\hline North & $302(19.6 \%)$ & $52(17.0 \%)$ & $354(19.2 \%)$ & \\
\hline East & $202(13.1 \%)$ & $31(10.2 \%)$ & $233(12.6 \%)$ & \\
\hline Northwest & $347(22.5 \%)$ & $50(16.4 \%)$ & $397(21.5 \%)$ & \\
\hline Southwest & $394(25.6 \%)$ & 97 (31.8\%) & $491(26.6 \%)$ & \\
\hline Northeast & $185(12.0 \%)$ & $61(20.0 \%)$ & $246(13.3 \%)$ & \\
\hline AKI stage & & & & $<0.001^{*}$ \\
\hline 1 & $988(64.2 \%)$ & $160(52.5 \%)$ & $1148(62.2 \%)$ & \\
\hline 2 & $304(19.7 \%)$ & $68(22.3 \%)$ & $372(20.2 \%)$ & \\
\hline 3 & $248(16.1 \%)$ & $77(25.2 \%)$ & $325(17.6 \%)$ & \\
\hline \multicolumn{5}{|l|}{ Underlying diseases } \\
\hline Hypertension & $639(41.5 \%)$ & $133(43.6 \%)$ & $772(41.8 \%)$ & 0.49 \\
\hline Coronary heart disease & $293(19.0 \%)$ & $68(22.3 \%)$ & $361(19.6 \%)$ & 0.19 \\
\hline Diabetes & $272(17.7 \%)$ & $57(18.7 \%)$ & $329(17.8 \%)$ & 0.67 \\
\hline Tumor & $223(14.5 \%)$ & $59(19.3 \%)$ & $282(15.3 \%)$ & $0.03^{*}$ \\
\hline Heart failure & $158(10.3 \%)$ & $40(13.1 \%)$ & $198(10.7 \%)$ & 0.14 \\
\hline CKD & $160(10.4 \%)$ & 27 (8.9\%) & $187(10.1 \%)$ & 0.42 \\
\hline Respiratory failure & $92(6.0 \%)$ & $55(18.0 \%)$ & $147(8.0 \%)$ & $<0.001^{*}$ \\
\hline COPD & $93(6.0 \%)$ & $28(9.2 \%)$ & $121(6.6 \%)$ & $0.04^{*}$ \\
\hline Hepatic cirrhosis & $74(4.8 \%)$ & $17(5.6 \%)$ & $91(4.9 \%)$ & 0.57 \\
\hline \multicolumn{5}{|l|}{ Concomitant diseases } \\
\hline Sepsis & $53(3.4 \%)$ & $20(6.6 \%)$ & $73(4.0 \%)$ & $<0.001^{*}$ \\
\hline MODS & $48(3.1 \%)$ & $34(11.1 \%)$ & $82(4.4 \%)$ & $0.01^{*}$ \\
\hline \multicolumn{5}{|l|}{ Injury factors } \\
\hline infection & $687(44.6 \%)$ & $172(56.4 \%)$ & $859(46.6 \%)$ & $<0.001^{*}$ \\
\hline medicine & $565(36.7 \%)$ & 98 (32.1\%) & $663(35.9 \%)$ & 0.13 \\
\hline Hypovolemia & 307 (19.9\%) & $73(23.9 \%)$ & $380(20.6 \%)$ & 0.12 \\
\hline Low cardiac output & $232(15.1 \%)$ & $77(25.2 \%)$ & $309(16.7 \%)$ & $<0.001^{*}$ \\
\hline Massive blood transfusion & 77 (5.0\%) & $14(4.6 \%)$ & $91(4.9 \%)$ & 0.76 \\
\hline Post-CPR & $41(2.7 \%)$ & $20(6.6 \%)$ & $61(3.3 \%)$ & $0.001^{*}$ \\
\hline DIC & $23(1.5 \%)$ & $13(4.3 \%)$ & $36(2.0 \%)$ & $0.001^{*}$ \\
\hline Rhabdomyolysis & $22(1.4 \%)$ & $2(0.7 \%)$ & $24(1.3 \%)$ & 0.42 \\
\hline Heart rate (bpm) & $82(76,98)$ & $87(80,105)$ & $84(76,99)$ & $<0.001^{*}$ \\
\hline BMI $\left(\mathrm{kg} / \mathrm{m}^{2}\right)$ & $23.6 \pm 3.7$ & $23.0 \pm 3.4$ & $23.5 \pm 3.6$ & $0.01^{*}$ \\
\hline MAP (mmHg) & $94.3 \pm 18.0$ & $92.0 \pm 21.5$ & $93.9 \pm 18.6$ & 0.10 \\
\hline Baseline SCr (mg/dl) & $1.04(0.85,1.42)$ & $1.06(0.79,1.45)$ & $1.04(0.83,1.42)$ & 0.63 \\
\hline \multicolumn{5}{|l|}{ Laboratory index } \\
\hline $\mathrm{Hb}(\mathrm{g} / \mathrm{dl})$ & $11.5 \pm 2.9$ & $11.2 \pm 3.0$ & $11.5 \pm 2.9$ & 0.11 \\
\hline WBC $\left(10^{9} / \mathrm{L}\right)$ & $9.2(6.4,13.6)$ & $11.5(7.9,17.9)$ & $9.6(6.6,14.2)$ & $<0.001^{*}$ \\
\hline PLT $\left(10^{9} / \mathrm{L}\right)$ & $172(109,234)$ & $148(80,217)$ & $167(104,232)$ & $<0.001^{*}$ \\
\hline $\mathrm{TP}$ (g/dl) & $6.2 \pm 1.2$ & $5.9 \pm 1.2$ & $6.2 \pm 1.2$ & $0.001^{*}$ \\
\hline $\operatorname{ALB}(\mathrm{g} / \mathrm{dl})$ & $3.4 \pm 0.8$ & $3.2 \pm 0.8$ & $3.3 \pm 0.8$ & $<0.001^{*}$ \\
\hline $\operatorname{ALT}(\mathrm{U} / \mathrm{L})$ & $22.0(13.2,45.0)$ & $31.5(17.0,77.5)$ & $23.6(14.0,50.9)$ & $<0.001^{*}$ \\
\hline AST (U/L) & $28.0(18.0,61.0)$ & $51.0(27.2,139.0)$ & $31.0(19.1,71.2)$ & $<0.001^{*}$ \\
\hline TBiL (mg/dl) & $0.8(0.5,1.4)$ & $1.0(0.6,2.1)$ & $0.8(0.5,1.5)$ & $<0.001^{*}$ \\
\hline DBiL (mg/dl) & $0.3(0.2,0.6)$ & $0.4(0.2,1.0)$ & $0.3(0.2,0.6)$ & $<0.001^{*}$ \\
\hline $\mathrm{SCr}(\mathrm{mg} / \mathrm{dl})$ & $1.7(1.3,2.6)$ & $1.8(1.4,3.1)$ & $1.8(1.3,2.7)$ & $0.01^{*}$ \\
\hline BUN (mg/dl) & $32.0(20.9,49.9)$ & $41.5(25.5,67.1)$ & $33.1(21.6,53.2)$ & $<0.001^{*}$ \\
\hline SUA $(\mathrm{mg} / \mathrm{dl})$ & $6.8(5.2,8.9)$ & $7.4(5.6,10)$ & $6.8(5.2,9.1)$ & $0.001^{*}$ \\
\hline Serum glucose (mg/dl) & $108.4(90.1,150.6)$ & $123.8(100.5,171.5)$ & $111.2(91.0,153.1)$ & $<0.001^{*}$ \\
\hline Serum potassium $(\mathrm{mmol} / \mathrm{L})$ & $4.1(3.7,4.5)$ & $4.2(3.7,4.9)$ & $4.1(3.7,4.6)$ & $0.04^{*}$ \\
\hline Serum calcium (mg/dl) & $8.6(8.0,9.2)$ & $8.3(7.6,8.9)$ & $8.6(7.9,9.1)$ & $<0.001^{*}$ \\
\hline Serum phosphate $(\mathrm{mg} / \mathrm{dl})$ & $3.8(3.0,4.7)$ & $3.9(2.9,5.6)$ & $3.8(3.0,4.8)$ & 0.17 \\
\hline Serum sodium (mmol/L) & $138.9(135,142)$ & $138.4(134.0,143.0)$ & $138.8(135,142)$ & 0.64 \\
\hline APACHE II score & $10(7,15)$ & $15(10,21)$ & $11(7,16)$ & $<0.001^{*}$ \\
\hline SOFA score & $5(2,8)$ & $8(4,11)$ & $5(2,9)$ & $<0.001^{*}$ \\
\hline AKI classification & & & & $0.004^{*}$ \\
\hline Pre-renal & $721(46.8 \%)$ & $161(52.8 \%)$ & $882(47.8 \%)$ & \\
\hline Intrinsic-renal & $659(42.8 \%)$ & $131(43.0 \%)$ & $790(42.8 \%)$ & \\
\hline Post-renal & $160(10.4 \%)$ & $13(4.3 \%)$ & $173(9.4 \%)$ & \\
\hline RRT & 115 (7.5\%) & $34(11.1 \%)$ & $149(8.1 \%)$ & $0.031^{*}$ \\
\hline Hospital stay (days) & $13(8,21)$ & $10(4,22)$ & $13(7,21)$ & $<0.001^{*}$ \\
\hline
\end{tabular}




\section{Kidney Blood Pressure Research}

Table 4. Percentage of drug-related AKI among the AKI patients

\begin{tabular}{ccccc}
\hline & Survivors $(\mathrm{n}=1540)$ & Non-Survivors $(\mathrm{n}=305)$ & Total $(\mathrm{n}=1845)$ & P value \\
\hline Drug related AKI & $220(14.3 \%)$ & $37(12.1 \%)$ & $257(13.9 \%)$ & 0.32 \\
Antibiotics & $103(6.7 \%)$ & $21(6.9 \%)$ & $124(6.7 \%)$ & 0.90 \\
ACEI/ARB & $40(2.6 \%)$ & $6(2.0 \%)$ & $46(2.5 \%)$ & 0.52 \\
anticancer drugs & $27(1.8 \%)$ & $5(1.6 \%)$ & $32(1.7 \%)$ & 0.89 \\
contrast medium & $29(1.9 \%)$ & $2(0.7 \%)$ & $31(1.7 \%)$ & 0.13 \\
Other drugs & $21(1.4 \%)$ & $3(1.0 \%)$ & $24(1.3 \%)$ & 0.78 \\
\hline \multicolumn{5}{c}{ Variables were expressed with n (\%). } \\
AKI, acute kidney injury AKI; ACEI/ARB, Angiotensin-Converting Enzyme Inhibitors/Angiotensin Receptor Blocker
\end{tabular}

Table 5. Risk factors associated with all-cause in-hospital mortality in AKI patients according to multivariate Cox regression analysis

\begin{tabular}{ccc}
\hline variables & HR $(95 \% \mathrm{CI})$ & P value \\
\hline Age (per 10 years increase) & $1.17(1.08,1.27)$ & $0.001^{*}$ \\
Tumor (yes vs no $\left.{ }^{*}\right)$ & $1.45(1.05,2.00)$ & $0.025^{*}$ \\
SUA (mg/dl) & $1.06(1.02,1.10)$ & $0.002^{*}$ \\
APACHE II score & $1.03(1.01,1.04)$ & $0.005^{*}$ \\
SOFA score & $1.05(1.02,1.09)$ & $0.003^{*}$ \\
\hline The variables with a P $<0.05$ in univariate Cox regression analysis were analyzed by multivariate Cox regression analysis. All variables \\
with a P $<0.05$ in multivariate Cox regression analysis were listed in the table and adjusted for regional distribution. \\
AKI, acute kidney injury; SUA, Serum uric acid; APACHE, Acute Physiology and Chronic Health Evaluation; SOFA, Sequential Organ Failure \\
\hline
\end{tabular}

patients underwent RRT, with intermittent hemodialysis (IHD), CRRT, SLED and others (i.e., hemoperfusion, and hemodiafiltration) accounting for 31.5\% (47 cases), 43.6\% (65 cases), $10.1 \%$ (15 cases) and $14.8 \%$ (22 cases), respectively.

\section{Mortality and Risk Factors in AKI Patients}

Regarding mortality, 305 AKI patients died during hospitalization; the total mortality rate was $16.5 \%$, which was higher than that of non-AKI patients $(0.6 \%, \mathrm{P}<0.001)$. The AKI patients had a longer hospitalization time $(13(7,21)$ days vs. $8(5,13)$ days, $\mathrm{P}<0.001)$ than the non-AKI hospitalized patients. Table 3 shows the epidemiological and clinical characteristics of the survivors and non-survivors among the AKI patients. The surviving patients were younger than those who died $(59.8 \pm 17.3$ vs. $64.5 \pm 17.7$ years, $\mathrm{P}<0.001)$. Of the non-survivors with AKI, $25.2 \%$ were at stage III, which was a higher proportion than that of the survivors (16.1\%). Except for hemoglobin, serum phosphorus, and serum sodium, all laboratory indicators differed significantly between these two groups. Serum uric acid (SUA) was used as a continuous variable in the Cox regression analysis. The APACHE II and SOFA scores of the non-survivors were higher than those of the survivors. The mortality rate of the patients who received RRT due to critical illness was significantly higher than that of the patients who did not. Among the AKI patients who received RRT, those who underwent CRRT had a higher mortality rate than those who underwent IHD, SLED, and other modalities. The mortality rates were $30.8 \%, 12.8 \%, 13.3 \%$ and $27.3 \%$, respectively. According to the univariate Cox regression analysis, the risk factors affecting the mortality of AKI patients included tumor, respiratory failure, COPD, MODS, sepsis, low cardiac output, disseminated intravascular coagulation (DIC), and post-resuscitation status. The multivariate Cox regression analysis showed that the independent factors affecting AKI patient mortality (Table 5) included age (increased by 10 years) (HR 1.17, 95\% CI 1.08-1.27), tumor (HR 1.45, 95\% CI 1.05-2.00), SUA (HR 1.06, 95\% CI 1.02-1.10), APACHE II score (HR 1.03, 95\% CI 1.011.04), and SOFA score (HR 1.05, 95\% CI 1.02-1.09). 


\section{Kidney Blood Pressure Research}

Risk Factor for Mortality among Laboratory Indicators: Serum Uric Acid

Among the laboratory indicators, SUA was the only independent predictor of mortality in the AKI patients. Figure 1 shows the different percentages of SUA among the survivors and non-survivors. The non-survivor group of AKI patients had a greater proportion of patients with a higher SUA level.

Furthermore, we transformed the SUA level into a categorical variable according to percentile, as follows: $\leq 25$ th percentile (SUA $\leq 5.2 \mathrm{mg} / \mathrm{dl}$ ), 26th-50th percentile $(5.2<$ SUA $\leq 6.8 \mathrm{mg} / \mathrm{dl}), 50$ th -75 th percentile $(6.8<$ SUA $\leq 9.1$ $\mathrm{mg} / \mathrm{dl}$ ), > 75th percentile (SUA $>9.1 \mathrm{mg} / \mathrm{dl}$ ). Table 6 shows the mortality for the different SUA groups. A Cox regression analysis was also conducted (Figure 2, Table 7); using SUA $\leq 5.2 \mathrm{mg} / \mathrm{dl}$ as the reference group, the HR for SUA $>9.1 \mathrm{mg} / \mathrm{dl}$ was 1.78 (95\%CI 1.23-2.58), that of $6.8<\mathrm{SUA} \leq 9.1 \mathrm{mg} / \mathrm{dl}$ was 1.40 (95\% CI 0.96-2.03) and that of $5.2<\mathrm{SUA} \leq 6.8 \mathrm{mg} / \mathrm{dl}$ was 1.11 (95\% CI 0.76-1.61).

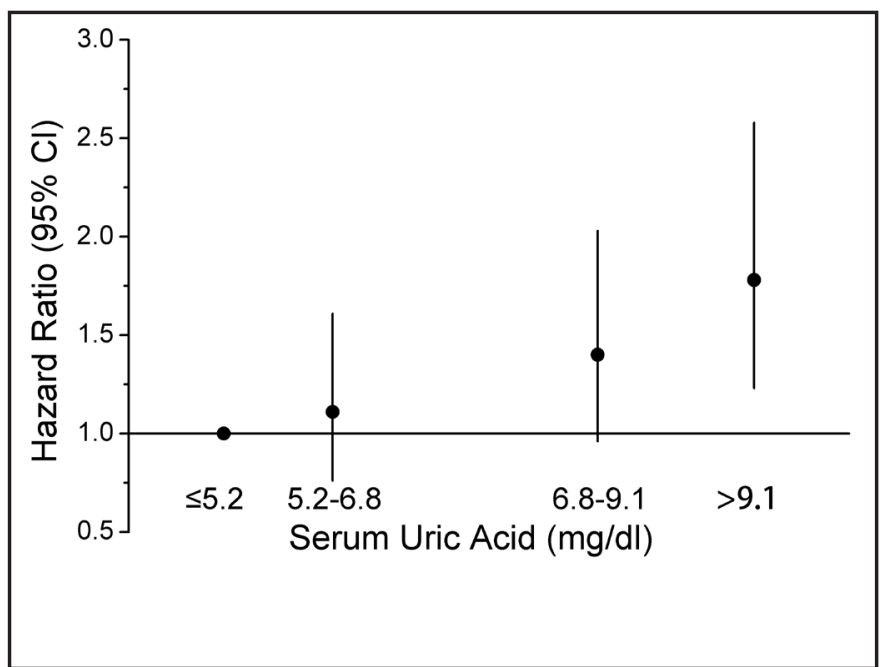

Fig. 2. Adjusted hazard ratio for mortality in all AKI patients with different serum uric acid levels. Adjusted for age, AKI stage, underlying disease, concomitant disease, damage factors, heart rate, BMI, region, laboratory indicators, APACHE II score, SOFA score, and RRT.

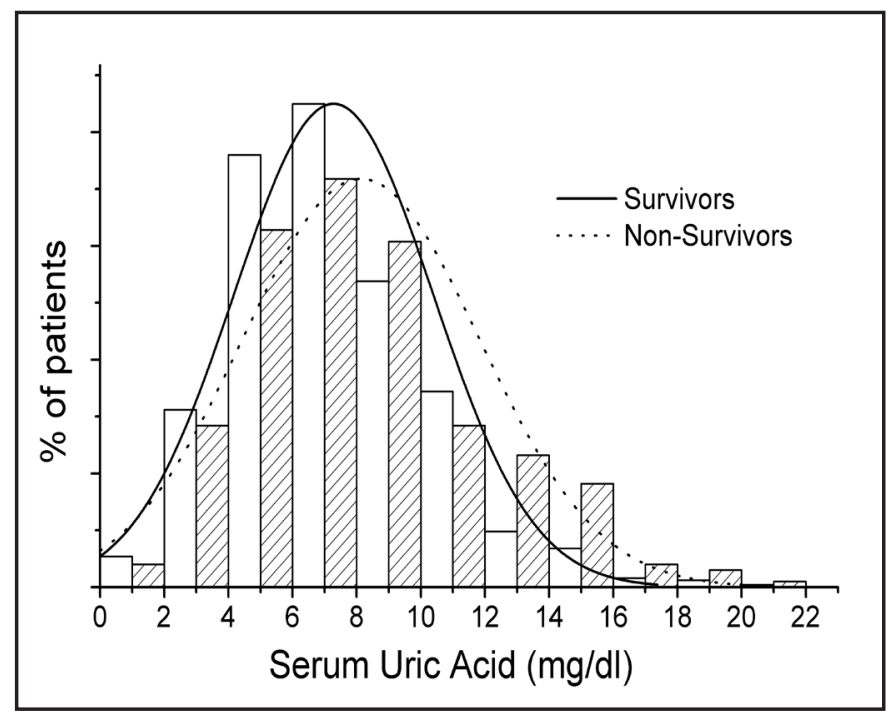

Fig. 1. Distribution of the proportions of serum uric acid concentrations in survivors and non-survivors.

Table 6. Mortality of different SUA groups

\begin{tabular}{cccccc}
\hline & Group 1 & Group 2 & Group 3 & Group 4 & Total \\
\hline SUA (mg/dl) & $\leq 5.2$ & $5.2-6.8$ & $6.8-9.1$ & $>9.1$ & \\
Cases & 455 & 452 & 472 & 466 & 1845 \\
Mortality (\%) & 14.2 & 14.5 & 15.2 & 22.1 & 16.5 \\
\hline \multicolumn{5}{c}{ SUA, Serum uric acid } \\
\hline \multicolumn{6}{c}{}
\end{tabular}

After the propensity score analysis, a total of 294 pairs of AKI patients with different survival conditions were included in the matched group set. There were no differences in demographic data, medical history, baseline renal function, and disease severity in the set. SUA > $9.1 \mathrm{mg} / \mathrm{dl}$ (HR1.73, 95\%CI 1.24-2.42) was still an independent risk factor for mortality in the multivariate Cox analysis for the matched sample set.

\section{Discussion}

AKI itself is not a single disease, rather, it is a clinical syndrome with complex 


\section{Kidney Blood Pressure Research}

Table 7. Risk factors associated with all-cause in-hospital mortality in AKI patients according to multivariate Cox regression analysis

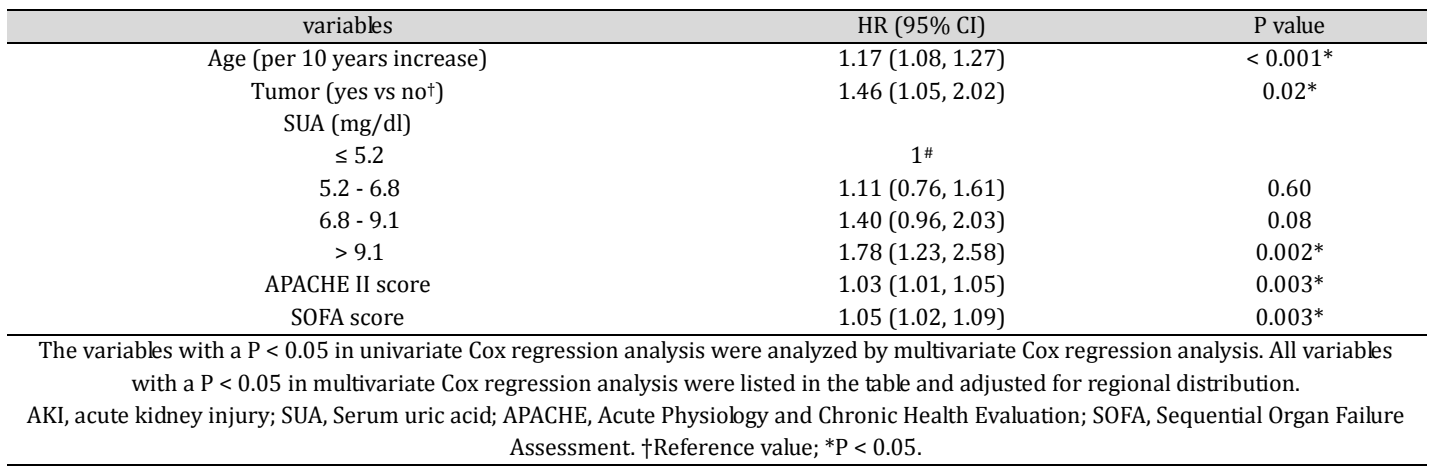

etiologies and high mortality [8]. Even a slight increase in creatinine will increase the possibility of a poor prognosis, by increasing the risk of mortality, progression to CKD or ESRD, or a cardiovascular event $[9,10]$. However, AKI is preventable and can even be reversed in early stages [1]. Indeed, if AKI is discovered and treated early and risk factors are removed as soon as possible, the progression of the disorder can be reduced, and patients' prognosis can be improved [1]. Therefore, it is very important to fully understand the risk factors and clinical characteristics of AKI's development and progression. However, non-traditional risk factors, especially laboratory indicators, tend to be neglected [11]. Accordingly, we designed a nationwide, multicenter study of AKI and selected representative hospitals, including some secondary hospitals in mainland China. Cases were collected according to the definition and diagnostic standards of the AKI clinical practice guidelines released in March 2012 to explore the risk factors, particularly laboratory indicators, that contribute to the development of AKI and a poor prognosis.

The AKI morbidity rate in this study was $0.97 \%$, which was consistent with previous reports in China [12] but significantly lower than the reported $21 \%$ for developed countries [1]. The main reason for this difference may be that AKI lacks specific symptoms, and its diagnosis is primarily based on changes in creatinine level. The lack of awareness of AKI in China result in a low frequency of serum creatinine measurements. The repeated creatinine assay rate in China ranges from $24.76 \%$ to $30 \%$ [12], which is much lower than the reported rate of $63.2 \%$ in developed countries [13]. Therefore, the prevalence of AKI in China is undoubtedly underestimated. Mehta et al [1]. reported a worldwide pooled incidence of AKI of $21 \%$ in a systematic review; however, this result was strongly dependent on the sampled population. Specialized populations, such as patients in intensive-care units (ICU), were included in the systematic review. Although some reports from several regions of Africa and Asia were included, the majority were from high-income countries, where populations were heavily weighted with ICU patients with high incidence and severity levels. Because of the incomplete Medical Grading System, a fair number of hospitalized patients in China are general patients with less severe disease, compared with those in developed countries, which will also result in a lower occurrence of AKI.

Many risk factors have been reported to be associated with the incidence of AKI, including age, female gender, CKD, diabetes, heart diseases, and lung diseases $[3,12,14]$. In accordance with previous studies, we found that advancing age, hypertension, and CKD were risk factors for AKI development. A large-scale prospective cohort study by Jiang et al [15]. indicated that male gender was an independent risk factor for cardiac surgery-related AKI, and a nationwide retrospective cohort study performed by Xue et al [16]. obtained the same conclusion. However, some large-scale observational studies on cardiac surgery [17], contrast agents [18], and aminoglycoside-related AKI [19] confirmed that female gender 


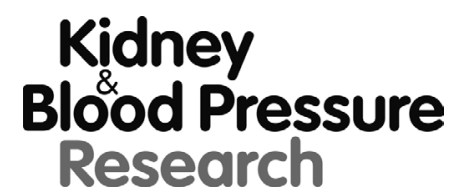

Kidney Blood Press Res 2017;42:761-773

\begin{tabular}{l|l}
\hline DOI: 10.1159/000484234 & ( 2017 The Author(s). Published by S. Karger AG, Basel \\
\hline
\end{tabular}

Nie et al.: Risk Factors for AKI Including Laboratory Indicators

was an independent risk factor for hospital-acquired AKI. Our study revealed that men had a greater risk of developing AKI than women, which was the same result as the former study. This issue remains controversial and requires further investigation.

The mortality rate of the AKI patients in our study was $16.5 \%$, consistent with other studies in China [20]. Most studies have consistently reported that the risk factors associated with a poor AKI prognosis include advancing age, cardiovascular disease, AKI stage, and so on [21]. In addition to examining these basic risk factors, we also systemically and comprehensively collected the laboratory indicators of patients with AKI, and examined the APACHE II and SOFA scores of these patients to better predict the mortality risk of AKI patients. The results showed that advancing age, tumor, high APACHE II and SOFA scores and high uric acid levels increased the mortality risk of hospitalized AKI patients. The APACHE II score is mainly used in ICUs to evaluate disease severity [22], whereas the SOFA score is used to evaluate the severity of functional failure of all organs [23]. Considering that AKI patients have high mortality, we used these two scores to evaluate all AKI patients, not only those in ICUs. The results showed that the mortality risks increased with the increase of the APACHE II or SOFA score. Similarly, two international multicenter prospective studies separated by 10 years confirmed a similar effect of the APACHE II score for predicting mortality risk [24, 25]. These results indicate that APACHE II and SOFA scores can accurately predict critical illness and mortality risk in AKI patients. The clinical value of these scores should be further examined and validated in studies with larger sample sizes.

Furthermore, we analyzed laboratory indicators and found that uric acid level was related to all-cause mortality. When analyzed as a dichotomous variable, the uric acid level and mortality risk of AKI patients displayed a J-shaped pattern. A propensity score came to the same conclusion. Considerable clinical evidence has suggested that elevated uric acid levels play an independent role in increased mortality, cardiovascular disease (CVD), and renal disease $[26,27]$. The relationship between serum uric acid and mortality risk in different populations, such as CKD patients [28], hemodialysis patients [29], peritoneal dialysis patients [30,31], and the general population [32], has been confirmed. High SUA has also been reported to be an independent predictor for AKI [33, 34]. In this study, we found that AKI patients with elevated serum uric acid concentrations had a higher inhospital mortality risk. The underlying mechanisms are discussed below, but must be explored further. Hyperuricemia results in endothelial dysfunction by inhibiting nitric oxide production [35]. Elevated SUA levels increase insulin resistance, dyslipidemia, hypertension and atherosclerosis. High SUA concentrations are significantly correlated with a decline in residual kidney function after adjusting for confounding factors [36]. Uric acid could have pro-inflammatory effects, increase oxidative stress, and damage the microcirculation [21], thereby increasing mortality risk and explaining why a high SUA level can serve as a marker of poor prognosis. Nevertheless, as high SUA might be a symptom of AKI, it is unclear whether the poor prognosis is due to uric acid itself or to AKI. Additionally, it remains unclear whether a reduction in uric acid could improve prognosis.

Although this study involved a nationwide epidemiological survey of AKI patients and collected many risk factors for AKI and prognostic factors for mortality, there are limitations. First, we did not perform long-term follow-up on AKI patients to evaluate the risk factors that affected their long-term prognosis. We have been conducting research in this area with AKI patients. Second, accurate measurement of urine output is so difficult for ordinary patients that most studies diagnose AKI only based on creatinine standards, as we did for this study. Furthermore, this multicenter study included AKI patients in general; therefore, the risk factors for some specific causes of AKI, such as contrast-induced AKI and AKI after cardiac surgery require further exploration. 


\section{Kidney Blood Pressure Research}

\section{Conclusion}

In summary, our study showed that the morbidity of hospitalized AKI patients in China was relatively lower than that in developed countries, though the mortality was the same. APACHE II and SOFA scores can be used to evaluate the in-hospital mortality risk of AKI patients. In addition to traditional risk factors, uric acid levels should be considered an independent predictor of all-cause mortality in AKI patients.

\section{Disclosure Statement}

The authors declare that they have no competing interests.

\section{Acknowledgements}

We are much obliged to Qingli Cai, Ying Liu, Ling Zhang, Haifeng Wang, Liyan Wang, Xuanyi Du, Huixia Cao, Shun Wang, Qinyun Li, Jinhong Xue, Peilin Dou, Zhanting Li, Zhimin Huang, Quanquan Shen, Rongrong Deng, Yue Cheng, Ruihua Wang, Jiuxu Bai, Yanqing Chi, Xinjun Yang, Caifeng Li, Jiefu Zhu, Fei Liu, Bo Li, Dandan Huang, Hongzhen Hu, Yuehua Gao and Xi Wang for their help with data collection. This study was supported by the Beijing Municipal Science and Technology Commission (Z131107002213011), the National Basic Research program of China (973 Program; no. 2015CB553605), the Special Fund for NHFPC Scientific Research in the Public Welfare (201502023) and the National Natural Science Foundation of China (81670694), the Fund of the Chinese PLA 12th Five-Year Plan for Medical Sciences (BWS14J040, BWS11J027).

\section{References}

1 Mehta RL, Cerdá J, Burdmann EA, Tonelli M, García-García G, Jha V, Susantitaphong P, Rocco M, Vanholder R, Sever MS, Cruz D, Jaber B, Lameire NH, Lombardi R, Lewington A, Feehally J, Finkelstein F, Levin N, Pannu N, Thomas B, Aronoff-Spencer E, Remuzzi G: International Society of Nephrology's 0by25 initiative for acute kidney injury (zero preventable deaths by 2025): a human rights case for nephrology. Lancet 2015;385:2616-2643.

2 Coca SG, Singanamala S, Parikh CR: Chronic kidney disease after acute kidney injury: a systematic review and meta-analysis. Kidney Int 2012;81:442-448.

3 Kidney Disease: Improving Global Outcomes (KDIGO) Group: KDIGO clinical practice guideline for acute kidney injury. Kidney Int Suppl 2012;2:1-138.

4 Levey AS, Eckardt KU, Tsukamoto Y, Levin A, Coresh J, Rossert J, De Zeeuw D, Hostetter TH, Lameire N, Eknoyan G: Definition and classification of chronic kidney disease: A position statement from Kidney Disease: Improving Global Outcomes (KDIGO). Kidney Int 2005;67:2089-2100.

5 Lio F, Pascual JL: Epidemiology of acute renal failure: A prospective, multicenter, community-based study. Kidney Int 1996;50:811-818.

6 Chang JW, Jeng MJ, Yang LY, Chen TJ, Chiang SC, Soong WJ, Wu KG, Lee YS, Wang HH, Yang CF, Tsai HL: The epidemiology and prognostic factors of mortality in critically ill children with acute kidney injury in Taiwan. Kidney Int 2015;87:632-639.

7 Kitchlu A, Adhikari N, Burns KEA, Friedrich JO, Garg AX, Klein D, Richardson RM, Wald R: Outcomes of sustained low efficiency dialysis versus continuous renal replacement therapy in critically ill adults with acute kidney injury: a cohort study. BMC Nephrol 2015;16:127.

8 Liu X, Guan Y, Xu S, Li Q, Sun Y, Han R, Jiang C: Early Predictors of Acute Kidney Injury: A Narrative Review. Kidney Blood Press Res 2016;41:680-700. 


\section{Kidney \\ Blood Pressure Research}

9 Lameire NH, Bagga A, Cruz D, De Maeseneer J, Endre Z, Kellum JA, Liu KD, Mehta RL, Pannu N, Van Biesen W, Vanholder R: Acute kidney injury: an increasing global concern. Lancet 2013;382:170-179.

10 Hu W, He W, Liu W, Fang X, Wu Y, Yu F, Hao W: Risk Factors and Prognosis of Cardiorenal Syndrome Type 1 in Elderly Chinese Patients: A Retrospective Observational Cohort Study. Kidney Blood Press Res 2016;41:672-679.

11 Funk I, Seibert E, Markau S, Girndt M: Clinical Course of Acute Kidney Injury in Elderly Individuals Above 80 Years. Kidney Blood Press Res 2016;41:947-955.

12 Yang L, Xing G, Wang L, Wu Y, Li S, Xu G, He Q Chen J, Chen M, Liu X, Zhu Z, Yang L, Lian X, Ding F, Li Y, Wang H, Wang J, Wang R, Mei C, Xu J, Li R, Cao J, Zhang L, Wang Y, Xu J, Bao B, Liu B, Chen H, Li S, Zha Y, Luo Q, Chen D, Shen Y, Liao Y, Zhang Z, Wang X, Zhang K, Liu L, Mao P, Guo C, Li J, Wang Z, Bai S, Shi S, Wang Y, Wang J, Liu Z, Wang F, Huang D, Wang S, Ge S, Shen Q, Zhang P, Wu L, Pan M, Zou X, Zhu P, Zhao J, Zhou M, Yang L, Hu W, Wang J, Liu B, Zhang T, Han J, Wen T, Zhao M, Wang H, ISN AKF 0by25 China Consortiums: Acute kidney injury in China: a cross-sectional survey. Acute kidney injury in China: a cross-sectional survey. Lancet 2015;386:1465-1471.

13 Zeng X, McMahon GM, Brunelli SM, Bates DW, Waikar SS: Incidence, outcomes, and comparisons across definitions of AKI in hospitalized individuals. Clin J Am Soc Nephrol 2014:9:12-20.

14 Anderson S, Eldadah B, Halter JB, Hazzard WR, Himmelfarb J, Horne FM, Kimmel PL, Molitoris BA, Murthy M, O’Hare AM, Schmader KE, High KP: Acute kidney injury in older adults. J Am Soc Nephrol 2011;22:2838.

15 Jiang W, Teng J, Xu J, Shen B, Wang Y, Fang Y, Zou Z, Jin J, Zhuang Y, Liu L, Luo Z, Wang C, Ding X: Dynamic Predictive Scores for Cardiac Surgery-Associated Acute Kidney Injury. J Am Heart Assoc 2016;5:e003754.

16 Xue JL, Daniels F, Star RA, Kimmel PL, Eggers PW, Molitoris BA, Himmelfarb J, Collins AJ: Incidence and mortality of acute renal failure in Medicare beneficiaries, 1992 to 2001. J Am Soc Nephrol 2006;17:11351142.

17 Thakar CV, Liangos O, Yared JP, Nelson D, Piedmonte MR, Hariachar S, Paganini EP: ARF after open-heart surgery: Influence of gender and race. Am J Kidney Dis 2003;41:742-751.

18 Mehran R, Aymong ED, Nikolsky E: A simple risk score for prediction of contrast-induced nephropathy after percutaneous coronary intervention. J Am Coll Cardiol 2004;44:1393-1399.

19 Smith CR, Moore RD, Lietman PS: Studies of risk factors for aminoglycoside nephrotoxicity. Am J Kidney Dis 1986;8:308-313.

20 Fang Y, Teng J, Ding X: Acute kidney injury in China. Hemodial Int 2014;19:2-10.

21 Varrier M, Ostermann M: Novel risk factors for acute kidney injury. Curr Opin Nephrol Hypertens 2014;23:560-569.

22 Knaus WA, Draper EA, Wagner DP, Zimmerman JE: APACHE II: a severity of disease classification system. Crit Care Med 1985;13:818-829.

23 Vincent JL, Moreno R, Takala J, Willatts S, De Mendonça A, Bruining H, Reinhart CK, Suter PM, Thijs LG: The SOFA (Sepsis-related Organ Failure Assessment) score to describe organ dysfunction/failure. Intens Care Med 1996;22:707-710.

24 Uchino S, Kellum JA, Bellomo R, Doig GS, Morimatsu H, Morgera S, Schetz M, Tan I, Bouman C, Macedo E, Gibney N, Tolwani A, Ronco C, Beginning and Ending Supportive Therapy for the Kidney (BEST Kidney) Investigators: Acute renal failure in critically ill patients: a multinational, multicenter study. JAMA 2005;294:813-818.

25 Bouchard J, Acharya A, Cerda J, Maccariello ER, Madarasu RC, Tolwani AJ, Liang X, Fu P, Liu ZH, Mehta RL: A Prospective International Multicenter Study of AKI in the Intensive Care Unit. Clin J Am Soc Nephro 2015;10:494-498.

26 Feig DI, Kang DH, Johnson RJ: Uric acid and cardiovascular risk. N Engl J Med 2008;359:1811-1821.

27 Nakagawa T, Kang DH, Feig D, Sanchez-Lozada LG, Srinivas TR, Sautin Y, Ejaz AA, Segal M, Johnson RJ: Unearthing uric acid: An ancient factor with recently found significance in renal and cardiovascular disease. Kidney Int 2006;69:1722-1725.

28 Madero M, Sarnak MJ, Wang X, Greene T, Beck GJ, Kusek JW, Collins AJ, Levey AS, Menon V: Uric acid and longterm outcomes in CKD. Am J Kidney Dis 2009;53:796-803.

29 Hsu SP, Pai MF, Peng YS, Chiang CK, Ho TI, Hung KY: Serum uric acid levels show a 'J-shaped' association with all-cause mortality in haemodialysis patients. Nephrol Dial Transpl 2004;19:457-462. 


\section{Kidney \\ Blood Pressure Research}

30 Xia X, He F, Wu X, Peng F, Huang F, Yu X: Relationship between serum uric acid and all-cause and cardiovascular mortality in patients treated with peritoneal dialysis. Am J Kidney Dis 2013;64:257-264.

31 Feng S, Jiang L, Shi Y, Shen H, Shi X, Jin D, Zeng Y, Wang Z: Uric Acid Levels and All-Cause Mortality in Peritoneal Dialysis Patients. Kidney Blood Press Res 2013;37:181-189.

32 Johnson RJ, Kang DH, Feig D, Kivlighn S, Kanellis J, Watanabe S, Tuttle KR, Rodriguez-Iturbe B, HerreraAcosta J, Mazzali M: Is there a pathogenetic role for uric acid in hypertension and cardiovascular and renal disease? Hypertension 2003;41:1183-1190.

33 Lee EH, Choi JH, Joung KW, Kim JY, Baek SH, Ji SM, Chin JH, Choi IC: Relationship between Serum Uric Acid Concentration and Acute Kidney Injury after Coronary Artery Bypass Surgery. J Korean Med Sci 2015;30:1509-1516.

34 Zuo T, Jiang L, Mao S, Liu X, Yin X, Guo L: Hyperuricemia and contrast-induced acute kidney injury: A systematic review and meta-analysis. Int J Cardiol 2016;224:286-294.

35 Khosla UM, Zharikov S, Finch JL, Nakagawa T, Roncal C, Mu W, Krotova K, Block ER, Prabhakar S, Johnson RJ. Hyperuricemia induces endothelial dysfunction. Kidney Int 2005;67:1739-1742.

36 Park JT, Kim DK, Chang TI, Kim HW, Chang JH, Park SY, Kim E, Kang SW, Han DS, Yoo TH: Uric acid is associated with the rate of residual renal function decline in peritoneal dialysis patients. Nephrol Dial Transplant 2009;24:3520-3525.

37 Nisula S, Kaukonen KM, Vaara ST, Korhonen AM, Poukkanen M, Karlsson S, Haapio M, Inkinen O, Parviainen I, Suojaranta-Ylinen R, Laurila JJ, Tenhunen J, Reinikainen M, Ala-Kokko T, Ruokonen E, Kuitunen A, Pettilä V, FINNAKI Study Group: Incidence, risk factors and 90-day mortality of patients with acute kidney injury in Finnish intensive care units: the FINNAKI study. Intens Care Med 2013;39:420-428. 